\title{
Valoración de un programa de educación alimentaria en pacientes con insuficiencia renal crónica en prediálisis
}

Evaluation of food education program in patients with chronic insufficiency in Pre-dialysis.

\author{
Autores \\ Melissa Lisbeth Ceròn Erazo. Universidad San Gregorio de Portoviejo. Manabi. Ecuador. \\ mlceron@sangregorio.edu.ec https://orcid.org/0000-0002-9837-2077
}

Carlos Sánchez Juan. Universidad de Valencia. Valencia. España. Carlos.Sanchez@uv.es https://orcid.org/0000-0002-0319-0722

Fecha de recibido: 2019-06-12

Fecha de aceptado para publicación: 2019-07-24

Fecha de publicación: 2019-09-30

\section{Resumen}

La Enfermedad Renal Crónica (ERC) tiene una prevalencia mundial de aproximadamente el 7,2\% en pacientes mayores de 30 años. La ERC cada día va en aumento en los países desarrollados, por lo que ofrecer una educación multidisciplinar a los pacientes en prediálisis puede retardar la progresión de la enfermedad, reducir la diálisis y tasa de mortalidad. El Objetivo de la investigación es valorar un programa de educación alimentaria en pacientes con Enfermedad Renal Crónica en fase de prediálisis. El enfoque es un estudio prospectivo observacional en la Escuela del Paciente Renal (EPR), fueron impartidos talleres de educación alimentaria. Se evaluaron medidas antropométricas, test de conocimientos al inicio y al final de la intervención. Luego de las actividades se observa mejora de los resultados del test sobre conocimientos tras la EPR. También aumentó la utilización de las técnicas de doble cocción. Y se observó un aumento del consumo de verduras y frutas con bajo contenido de potasio. Se concluye que, al realizar la EPR, los pacientes han aumentado sus conocimientos, mejorando sus hábitos alimentarios y técnicas culinarias, lo cual permite una repercusión positiva sobre el control de su enfermedad.

Palabras clave: Educación nutricional; escuela del paciente renal; hábitos saludables; insuficiencia renal crónica; prediálisis.

\begin{abstract}
Chronic Kidney Disease (CKD) has a worldwide prevalence of approximately $7.2 \%$ among patients over 30 years. CKD is increasing every day in developed countries; therefore, a multidisciplinary education in predialysis patients can delay disease progression, reduce of dialysis and mortality rates. The research objectives are to evaluate a food education program in patients with Chronic Kidney Disease in predialysis phase. The approach is a prospective observational study at the Kidney Patient School (KPS) food education workshops were taught Anthropometric knowledge tests measures were evaluated at the beginning and end of the intervention. After the activities, there is an improvement in the results of the knowledge test after the KPS. There was an increment in the use of double cooking techniques. And was detected an increase in the consumption of vegetables and fruits with low potassium content. It is concluded that the KPS. Patients have increased their knowledge, improving their eating habits and culinary techniques. Which allows a positive impact on the control of their disease.
\end{abstract}

Keywords: Chronic kidney diseases. healthy habits. kidney patient school. nutritional education. predialysis. 


\section{Introducción}

Los riñones son órganos encargados de realizar funciones vitales para nuestro organismo, como son las funciones excretoras, reguladoras y endocrinas. Basado en estos fundamentos al hablar de un fallo renal, (Martìnez-Castelao et al., 2014) define la presencia de una enfermedad renal crónica (ERC) utilizando las directrices de las actuales guías Kidney Disease Improving Global Outcomes (KDIGO) que establece entre sus criterios de diagnóstico a la tasa de filtrado glomerular (TFG) inferior a $60 \mathrm{ml} / \mathrm{min} / 1,73 \mathrm{~m} 2$ (durante al menos 3 meses) o a la existencia de una lesión renal demostrada de forma directa en una biopsia renal o indirecta mediante el incremento de albuminuria, o cuando hay presencia de alteraciones en el sedimento de orina .

Tomando en cuenta el filtrado glomerular (FG) el grado de albuminuria y la etiología. Los grados de FG (G1 a G5) y de albuminuria (A1 a A3) se exponen en la Tabla 1 (ver en Anexos).

Según criterio de (Salas, Bonada, Trallero \& Salò, 2000) en su estudio, "La enfermedad renal crónica se caracteriza por la acumulación de una serie de sustancias en la sangre, ya sea por descenso en su excreción, aumento de su producción, menor degradación renal, o más bien por alteración en su metabolismo" (pp. 232- 233).

Existe una prevalencia media mundial de aproximadamente el 7,2\% en pacientes mayores de 30 años con ERC. Se estima que en España alrededor del 9,2\% de la población adulta sufren dicha enfermedad, con una prevalencia global en los estadios 3-5 del 6,8\%, elevándose este último porcentaje al 20,6\% en sujetos mayores de 64 años. (Otero, Francisco, Gayoso, \& Garcia, 2010, pp. 78- 86).

Muchos estudios confirman (Caro \& Teruel, 2010); (Walker, Wagner, \& Tangri, 2014) una elevada prevalencia, con proyección a incrementar en los próximos años, la ERC se asocia con eventos adversos clínicos y funcionales, así como con una importante morbimortalidad cardiovascular.

La ERC usualmente se empeora muy lentamente, y puede ser que los síntomas no se enseñen hasta que los riñones están muy dañados. Se puede presentar diversos síntomas que son causados por la acumulación de deshechos y liquido extra en tu cuerpo: Prurito, calambres musculares, náuseas o vómitos entre otras manifestaciones (Fund, 2019).

Es por ello, "fundamental un adecuado e individualizado tratamiento nutricional en los pacientes con IRC en prediálisis para mantener un peso saludable y evitar la desnutrición, requiriendo una ingesta energética de 30-35 kcal/kg/día" según las guías (National Kidney Foundation, 2002, p. 226).

Por otra parte, para enlentecer la progresión de la nefropatía a su fase terminal y limitar la producción de toxinas urémicas, estas directrices recomiendan a las personas con IRC en fase de pre-diálisis, la institución de una dieta baja en proteínas, concretamente de $0,60 \mathrm{~g} / \mathrm{kg} / \mathrm{día}$; y para aquellas personas que no aceptan este tipo de dieta o que son incapaces de mantenerla adecuadamente, puede ser prescrito un consumo de hasta $0,75 \mathrm{~g} / \mathrm{kg} /$ día de proteínas. Esto es a causa de que la acumulación en sangre de productos nitrogenados derivados del metabolismo de proteínas genera anorexia, náuseas y trastornos gastrointestinales que impiden una adecuada ingesta (Alcàzar Arroyo et al. , 2008). 
Dicha ingesta de proteína debe ser de valor biológico elevado, un 75\% del total, para asegurar una adecuada ingesta y fuentes de aminoácidos ya que la ingesta se ve relacionada directamente en las calorías totales que son de vital importancia en el paciente renal para evitar el catabolismo proteico corporal (Perberton, 1993, pp. 226 - 228).

El riñón es el principal regulador del balance de sodio y potasio en los pacientes con IRC y esta función se ve comprometida, por lo cual hay una retención de dichos minerales alcanzando altos niveles de toxicidad. Por este motivo hay altas probabilidades de desarrollar hipertensión, hiperfosfatemia. Según (Beto, Schury, \& Bansal, 2016) entre un 20 a un $30 \%$ de los pacientes con ERC presentan pobre adherencia a la dieta y el tratamiento. En varios estudios cualitativos llevados a cabo en la ERC para identificar las principales barreras a la adherencia en el consumo de sodio los temas recurrentes fueron el "autocontrol" del consumo y el reconocimiento de sodio oculto en alimentos procesados.

Restringiendo su consumo se evitaría complicaciones secundarias como riesgos cardiovasculares y caída del filtrado glomerular. Recomiendan (de Luis Ramòn \& Bustamante, 2008) el remojo y técnicas de cocción de verduras y legumbres que conlleven a la eliminación del agua de cocción, ya que se ha demostrado que pueden disminuir hasta en un $40 \%$ su contenido en potasio.

Es de gran importancia que el paciente se involucre en el manejo de su tratamiento, tanto en las medidas higiénicas y dietéticas, para reducir complicaciones, siendo por ello fundamental la educación sanitaria y los grupos de apoyo donde se le brinde al paciente un cuidado integral que contemple información y formación educacional. Es por esto que en el Hospital General Universitario de Valencia se ha conformado desde el 2011 un programa denominado "Escuela del Paciente Renal", dirigida por un equipo multidisciplinar (equipo de nefrología, endocrinología, nutrición, enfermería y psicología) con el objeto de ser un medio de apoyo para los pacientes que presentan esta enfermedad, promoviendo por métodos educativos y charlas en equipo el conocimiento sobre la patología que presentan.

Según Yu et al. (2014) "La educación multidisciplinar en los pacientes en prediálisis retarda la progresión de la enfermedad, reduce la incidencia de la diálisis, la mortalidad y también se ha observado un ahorro económico en los servicios de hospitalización” (p. 6).

Entre las distintas estrategias de educación, la formación grupal presenta diversas ventajas. Por un lado, se ha demostrado la utilidad de resolver dentro del grupo las inquietudes y problemas que le surjan a un paciente, además, es muy positiva la interacción con pacientes que actúan como mentores explicando su propia experiencia, pudiendo proporcionar a los nuevos pacientes con IRC la comprensión y los conocimientos derivados de sus vivencias (Aguilera Flores et al., 2012).

En cuanto a la educación alimentaria, su objetivo principal es darle poder y conocimientos al paciente para que éste pueda por sí solo sobrellevar sus necesidades adaptada a la etapa de su enfermedad, sin la necesidad de la presencia a largo plazo de un profesional. La utilización de material educativo y la realización de talleres o actividades grupales ayudan a motivar y mejorar el cumplimiento de las pautas dietéticas.

En marzo del 2003, en Zurich, Suiza, tuvo lugar una conferencia de consenso en la que trataron las normas de calidad para una educación dirigida a pacientes con IRC en prediálisis. En dicha conferencia, se llegó a la conclusión de que existe una creciente evidencia de la 
importancia de la educación dirigida por un equipo multidisciplinar (enfermeras, nefrólogos, psicólogos, dietistas-nutricionistas, etc.) como opción de terapia para pacientes con IRC, ya que puede dar lugar a una mayor calidad de vida, a la mejora de los resultados clínicos, una tasa de supervivencia mayor, menor incidencia de mortalidad debida a un evento cardiovascular después del inicio de la diálisis y la reducción de los costos de atención sanitaria, ya que se ha evidenciado una reducción del tiempo de hospitalización y de la necesidad de entrada a diálisis de manera urgente. Sin embargo, todavía no hay una estandarización sobre la forma óptima para diseñar o ejecutar dichos programas. (Maffei, Savoldi \& Triolo, 2013); (Isnard et al. , 2014).

\section{Objetivo}

Valorar un programa de educación alimentaria y la influencia que tiene en la salud de los pacientes con Enfermedad Renal Crónica en fase de prediálisis.

\section{Objetivos específicos}

Valorar los cambios alimentarios tras el programa.

Determinar la mejoría en conocimientos alimenticios y nutricionales.

\section{Metodología}

Estudio prospectivo observacional en 61 pacientes ambulatorios con enfermedad renal crónica en prediálisis que participan en la "Escuela del Paciente Renal" (EPR) del Consorcio Hospital General Universitario de Valencia.

Los criterios de inclusión para la selección de los pacientes participantes fueron los siguientes: IRC en estadio $\geq 3\left(\mathrm{FG}<60 \mathrm{ml} / \mathrm{min} / 1,73 \mathrm{~m}^{2}\right)$, que sean mayores de 18 años, con disponibilidad y deseo de querer participar en los talleres. Se excluyeron a los pacientes que ya están sometidos a diálisis.

A cada participante se le explicó la naturaleza y propósito del estudio, obteniendo de todos ellos el consentimiento informado, en función de la legislación vigente (Ley 41/2002) y tras previa evaluación y aprobación por el Comité de Investigación y Ética de la Universidad de Valencia.

El programa dirigido por el personal sanitario del Hospital General Universitario de Valencia (equipo de nefrología, endocrinología, nutrición, enfermería y psicología) cumplieron en cada edición de la escuela, 6 sesiones de 2 horas de duración y con frecuencia semanal a grupos de 6-10 personas (con la posibilidad de ser acompañados por algún familiar) en las que una Dietista-Nutricionista y un Psicólogo impartieron talleres de educación alimentaria y apoyo psicológico, respectivamente, enfocado a las necesidades requeridas para personas con IRC no sometidas a tratamiento renal sustitutivo.

En la primera sesión se realizaron medidas antropométricas (talla, peso, IMC, \% masa grasa, \% masa muscular, grasa visceral y metabolismo basal) mediante tallímetro de precisión milimétrica y bioimpedanciómetro Omron BF511. Por otro lado, se les facilitó un test de 24 preguntas tipo verdadero/falso sobre conocimientos relacionados con la alimentación en general y el tratamiento nutricional durante la enfermedad renal crónica y un cuestionario de frecuencia de consumo de los alimentos elaborado por el equipo de nefrología y nutrición del hospital, que se ha utilizado en anteriores ediciones de la escuela renal. 
En las 4 siguientes sesiones, en los talleres de alimentación y nutrición, mediante presentaciones PowerPoint y fichas educativas de manera activa y participativa; se explicó a los pacientes los siguientes temas: sistema renal y nutrientes; objetivos del tratamiento nutricional y alimentación del paciente en prediálisis; consejos dietéticos y culinarios y recetas de cocina adaptadas a la enfermedad renal crónica; y, por último, etiquetado nutricional y mitos de alimentación. En cuanto a los talleres de psicología, los temas tratados fueron los siguientes: control del pensamiento, entrenamiento en toma de decisión, ansiedad y depresión; trastornos del sueño; y habilidades de comunicación y asertividad.

En la última sesión se realizaron medidas antropométricas (pliegue tricipital, circunferencia de cadera y circunferencia braquial) utilizando plicómetro Holtain de 0,2 $\mathrm{mm}$ de sensibilidad y cinta métrica inextensible de precisión milimétrica, con el fin de recoger más datos que caracterizaran a los pacientes. También se les volvió a entregar el test de conocimientos y el CFC (Cuestionario de Frecuencia de Consumo alimentario) de alimentos para poder evaluar si se consiguió el objetivo de mejorar los conocimientos nutricionales adquiridos tras las sesiones educativas.

Los datos descriptivos fueron introducidos en un documento de Excel; los cuantitativos se muestran como media y desviación estándar $(\mathrm{X} \pm \mathrm{DS})$ y los cualitativos como frecuencias y porcentajes (\%). Para la comparación de datos en antes-después se utilizó el programa estadístico SPSS 20.0, con el que se realizó la T-student para aquellos datos que seguían una normalidad, y como pruebas no paramétricas se utilizaron el test de Wilcoxon para los datos cuantitativos y el test de McNemar para los cualitativos. Se consideró como diferencia estadísticamente significativa aquella con un valor $\mathrm{p}<0,05$ (probabilidad superior al 5\%).

\section{Resultados}

Para la realización del estudio se llevaron a cabo 9 ediciones de la escuela del paciente renal, en la que participaron un total de 61 participantes, de los cuales el $67,2 \%$ ( $n=41$ ) eran hombres y el 32,8\% $(n=20)$ eran mujeres, con una edad media de 65,89 $\pm 12,62$ años.

Para la evaluación del nivel de conocimientos fueron analizados 54 cuestionarios, puesto que 7 pacientes faltaron a la última sesión y no realizaron el cuestionario final. Como se pudo apreciar, antes de la educación alimentaria grupal se obtuvo una media de 15,69 preguntas acertadas $(67 \%)$ y 8,31 preguntas falladas (33\%); mientras que al final de la intervención 19,69 preguntas fueron acertadas $(82,04 \%)$ y solo 4,31 preguntas fueron falladas $(17,96 \%)$. Se demuestra por tanto una mejora de los conocimientos tras la intervención con educación alimentaria con significación estadística (test de Wilconxon, $\mathrm{p}<0,001$ ). Ver Figura 1 (en Anexos).

Antes de impartir las charlas de educación alimentaria, la pregunta con un mayor número de fallos fue la $\mathrm{n}^{\circ} 21$ que describía: Las hierbas aromáticas y las especias no están recomendadas en la insuficiencia renal. El número de fallos de esta pregunta fue de 41 y paso a 26 en el test final. Otras preguntas con un número alto de fallos al inicio fueron la $n^{\circ} 11$ (En la insuficiencia renal está muy aconsejado el consumo de alimentos ricos en fósforo) y la $\mathrm{n}^{\mathrm{o}} 17$ (Los alimentos de origen vegetal no tienen proteínas).

En cuanto a las preguntas con un mayor número de aciertos tras la intervención educativa destacan: la $\mathrm{n}^{\circ} 6$ (La cebolla, el pepino y el pimiento son verduras con un contenido pobre en potasio) y en $\mathrm{n}^{\mathrm{o}} 15$ (El remojo y la doble cocción son dos técnicas culinarias que se utilizan para 
disminuir el contenido de potasio en los alimentos) con 9 fallos y la $\mathrm{n}^{\mathrm{0}} 13$ (En la insuficiencia renal está muy recomendado el consumo de verdura congelada, pues contiene una menor cantidad de potasio) con 7 fallos.

Las preguntas en las que se obtuvo una mayor diferencia de aciertos entre el test inicial y el final y por tanto muestran un mayor aprendizaje fueron la $n^{\circ} 11$, la $n^{\circ} 13$ y la $n^{\circ} 23$ (Los productos integrales están muy recomendados en la insuficiencia renal).

También se valoró el aprendizaje de los pacientes sobre las técnicas culinarias recomendadas en IRC para disminuir el contenido de potasio de los alimentos: remojo y doble cocción. En la figura se muestran los porcentajes de pacientes que utilizaban estas técnicas antes de la asistencia a la EPR y los que las utilizaban después. Tanto para la técnica de doble cocción como para la técnica del remojo hay una mejora del conocimiento de la misma con significación estadística (ambos $\mathrm{p}<0,001)$. También se observa un aumento de uso con significación estadística para ambas técnicas $(\mathrm{p}=0,002)$.

En cuanto al cuestionario de Frecuencia de Consumo de alimentos que se les paso a los pacientes antes y después de los talleres se observaron los siguientes cambios:

Proteínas: La recomendación en cuanto al consumo de alimentos proteicos en pacientes con IRC debe estar controlada la ingesta proteica y también porque son alimentos ricos en fosforo. El consumo de alimentos proteicos se mantiene (1,50 veces al día antes y 1,43 veces al día después de la educación alimentaria) y las recomendaciones en las legumbres es reducir su consumo a una vez a la semana. En este caso, tras la EPR, se reduce sin haber una gran diferencia, de 1,73 a 1,56 veces a la semana $(\mathrm{p}=0,150)$.

Cereales: cabe destacar la reducción del consumo de pan integral, no recomendado en este colectivo por la cantidad de fósforo que contiene. El consumo pasa de 2,64 a 0,06 veces a la semana, reducción estadísticamente significativa $(\mathrm{p}=0,002)$.

Frutas y verdura: con alto contenido en potasio y con bajo contenido en potasio. En el caso de las frutas con alto contenido en potasio, se redujo el consumo de todas ellas: plátano, de 1,19 a 0,24 veces/semana $(p<0,001)$; kiwi de 0,53 a 0,23 veces/semana $(p=0,281)$ y uva de 1,73 a 0,72 veces/ semana $(p=0,43)$. En cambio, las frutas con bajo contenido en potasio vieron aumentado su consumo, por ejemplo, la fresa que pasa de 1,47 a 1,92 veces a la semana $(p=0,041)$. Lo mismo se observa en el caso de las verduras: se muestra un aumento del consumo de verduras con bajo contenido en potasio, a destacar el pimiento ( 2,15 vs. 2,75 veces/semana; $p=0,026)$ por

ser estadísticamente significativo. Se reduce el consumo de acelgas y espinacas, verduras con alto contenido en potasio ( 0,81 vs. 0,61 veces/semana; $p=0,164$ y 0,66 vs. 0,43 veces/semana; $\mathrm{p}=0,105$ respectivamente).

Agua: la recomendación en IRC es beber agua de mineralización débil. Tras la EPC, el $63 \%(n=34)$ de los pacientes consumen esta agua frente al 50\% $(n=29)$ del inicio $(\mathrm{p}=0,057)$. Solo el 13\% $(n=7)$ continúa tomando agua del grifo.

\section{Discusión}

Según Aguilera Flores et al. (2012) la atención a pacientes con ERC por un Equipo Multidisciplinar es una estrategia sugerida hace ya más de un decenio por grupos canadienses. Al tratarse con este equipo se demuestra un mejor conocimiento de la ERC y mejores parámetros bioquímicos. 
Con la ayuda de dicho equipo se facilita el acceso a una información más detallada y balanceada, proporciona un cuidado más eficiente, efectivo y global tanto de los pacientes como de sus familiares, enlentece la progresión de la ERC, disminuye la comorbilidad y las complicaciones.

Existen muchas publicaciones relacionadas con la ERC basada en el autocuidado, en ofrecer más conocimientos sobre su enfermedad y en empoderar al paciente en las diferentes terapias sustitutivas hemodiálisis y diálisis peritoneal, para poder facilitarle la opción posterior a elegir del tratamiento. Sin embargo, se encontró muy poca bibliografía que engloba el estado nutricional, el patrón alimentario, estado de ansiedad y grado de satisfacción con la educación recibida.

Tras una intervención nutricional de 6 meses con un PIN (Programa de intervención nutricional) en pacientes con ERC, se observó una mejora de su estado nutricional, incremento del porcentaje de pacientes que cumplían criterios de normonutrición $(58,0$ vs $80,0 \%)$ asociada a una disminución del porcentaje de malnutridos (28,0 vs 8,0\%), mejora de la calidad de la dieta, así como un mejor control del estado antropométrico del colectivo (Pèrez Torres et al. 2013).

Todo lo mencionado ayuda argumentar a este estudio, que el mejorar los conocimientos en alimentaciòn en los pacientes con enfermedad renal, repercute de manera positiva en su estado nutricional y calidad de vida.

Por otro parte, otros autores observaron que una intervención educativa sencilla puede mejorar el conocimiento de la ERC entre la población general añosa, más susceptible de padecerla. Y mostró como resultado que, tras la intervención educativa, un $80 \%$ de los individuos participantes mejoraron significativamente la puntuación del cuestionario de conocimientos sobre la ERC (Bote i Fernandèz, 2009).

Según Benini et al. (2018) refieren el concepto de adherencia terapéutica como el grado en que el comportamiento de una persona (tomar medicamentos seguir un régimen alimentario y ejecutar cambios del modo de vida) se define como el compromiso de colaboración activa e intencionada por parte del paciente con el fin de producir el resultado preventivo y terapéutico deseado.

De la misma manera, Aguilera Flores et al. (2012) en su estudio de "Educación en grupo y multidisciplinar en pacientes renales y sus familiares", ha demostrado una alta satisfacción con la experiencia de esta intervención. No obstante, describió en la mayoría un ligero aumento de ansiedad; lo cual, lo interpreta como útil en el sentido que les permite movilizarse para cambiar pautas de alimentación; lo que resulta interesante para futuras investigaciones. Y desde luego se demostró un efecto positivo y un aumento de sus conocimientos sobre ERCA y técnicas de sustitución.

De este modo Yu et al. (2014) describe que la alta prevalencia de enfermedad renal terminal constituye una gran carga económica para el paciente, la sociedad y el país, exigiendo una alta utilización de los gastos médicos y de atención de la salud y concluye que con la educación prediálisis puede disminuir la incidencia de enfermedad renal terminal y la mortalidad en el primer año de la diálisis. 


\section{Conclusiones}

Un programa de educación alimentaria en paciente con ERC en fase de prediálisis ayuda a mostrar mejoría en los conocimientos sobre la alimentación más adecuada y el tratamiento nutricional de su enfermedad.

Después de realizar las intervenciones del programa de educación alimentaria se pudieron observar cambios en el consumo de alimentos e implementación de técnicas culinarias para reducir el potasio.

Una vez finalizados los talleres de educación alimentaria se observó un mayor número de aciertos en las respuestas acerca de los alimentos más adecuados de consumo en el tratamiento nutricional en la ERC.

\section{Referencias Bibliográficas}

Aguilera Flores , A., Prieto Velasco , M., Gonzàlez Romero, L., Abad Toral , B., Martìnez Crespo , E., Robles del Rìo, I., \& Boso Serrano , P. (2012). Una estrategia poco utiliizada en el cuidado de pacientes con enfermedad renal cronica: La educacion en grupo multidisciplinar de pacientes y sus familiares. Enfermeria Nefrologia, 15(1), 14-21.

Alcàzar Arroyo, R., Orte, L., Gonzalez Parra, E., Gòrriz, J., Navarro, J., Martìn de Francisco, A., .. . Àlvarez Guisasola, F. (2008). Documento de SEN-semFYC sobre la enfermedad renal crònica. Nefrologìa, 28(3), 273-282.

Benini, R., Cruz , P., Linari , M., Szlain, P., Zelechower, H., \& Zlotnitzky, V. (2018). ESTUDIO DE INTERVENCIÓN EDUCATIVA EN PACIENTES CON ENFERMEDAD RENAL ESTADIO 5D SOBRE TENSIÓN ARTERIAL Y SU RELACIÓN CON LA INGESTA DE SODIO. Actualización en Nutrición, 19(1), 12-21.

Beto, J., Schury, K., \& Bansal, V. (2016). Stategies to promote adherence to nutritional advice in patients with chronic kidney disease: a narrative review and commentary. International Journal of Nephrology and Renovascular Disease, 9, 21 -33.

Bote i Fernandèz, C. (2009). Intervención educativa sobre la enfermedad renal crònica en atenciòn primaria. Sociedad Española de Enfermeria Nefrologia, 12(4), 90-92.

Caro, J., \& Teruel, J. (2010). Importancia de la detección temprana de la enfermedad renal crònica en grupos de riesgo. Medicina Clinica (Barc), 134(6), 257-259.

de Luis Romàn , D., \& Bustamante, J. (2008). Aspectos nutricionales en la insuficiencia renal. Nefrologìa, 28(3), 333-342.

Fund, A. K. (2019). Kidneyfund.org. Obtenido de http://www.kidneyfund.org/en-espanol/enfermedad-delos-rinones/falla-de-los-rinones/. 
Gorostidi, M., Santamarìa, R., Alcàzar, R., Fernàndez-Fresnedo, G., Galceràn, J., Goicoechea, M., .. . Ruilope, L. (2014). Documento de la Sociedad Española de Nefrologìa sobre las guias KDIGO para la evaluaciòn y tratamiento de la enfermedad renal crònica. Nefrología (Madrid), 3, 302-316.

Isnard Bagnis , C., Crepaldi, C., Dean, J., Goovaerts, T., Melander, S., Nilsson, E., . . Mooney, A. (2014). Quality standards for predialysis education: Results from a consensus conference. Nephrology Dialysis Transplantation, 30(7), 1058-1066.

Maffei, S., Savoldi, S., \& Triolo, G. (2013). When should commence dialysis: Focusing on the predialysis condition. Nephrourology Monthly, 5(2), 723.

Martìnez-Castelao, A., Gòrriz, J., Bover, J., Segura-de la Morena, J., Cebollada, J., Escalada , J., . . . Hernàndez- Moreno, J. (2014). Documento de consenso para la detecciòn y manejo de la enfermedad renal crònica. Endocrinologìa y Nutriciòn, 61(9), e25-e43.

National Kidney Foundation, K. (2002). Clinical practice guidelines for chronic kidney disease: evaluation, classification and stratification. Am J Kidney Dis, 38(1), 266.

Otero Gonzàlez, A., De Francisco , A., Gayoso, P., Garcìa, F., \& EPIRCE Study Group. (2010). Prevalence of chronic renal disease in Spain: Results of the EPIRCE study. Nefrologia, 30(1), 7886.

Perberton, C. (1993). Manual de Dietètica de la Clinica de Mayo (6 ed.). Barcelona: Mèdicini.

Pèrez Torres, A., Gonzàlez , E., Bajo , M., Palma Milla, S., Sànchez Villanueva, R., Bermejo, L., .. . Grupo de nutriciòn renal de la Paz. (2013). Evaluación de un programa de Intervención Nutricional en pacientes con enfermedad renal cronica avanzada (ERCA). Nutriciòn Hospitalaria, 28(6), 2252-2260.

Salas , J., Bonada, A., Trallero , R., \& Salò, M. (2000). Nutriciòn y Dietètica Clìnica. Barcelona: Masson.

Walker, S., Wagner, M., \& Tangri, N. (2014). Chronic kidney disease, frailty, and unsuccessful aginf: a review. Journal of Renal Nutrition, 24(6), 364-370.

Yu, Y., Wu, I., Huang, C., Hsu, K., Lee, C., Sun, C., . . Wu, M. (2014). Multidisciplinary predialysis education reduced the inpatient and total medical costs of the first 6 months of dialysis in incident hemodialysis patients. Plos One, 9(11), e112820. 
Anexos

Tabla 1: Clasificación de la ERC.

Fuente: Gorostidi et al.(2014) Clasificación de ERC.

\section{Categorías del FG}

Categoría FG (ml/min) Descripción:

\begin{tabular}{lll}
\hline G1 & $\geq 90$ & Normal o elevado \\
G2 & $60-89$ & Ligeramente disminuido \\
G3a & $45-59$ & Ligera a moderadamente disminuido \\
G3b & $30-44$ & Moderada a gravemente disminuido \\
G4 & $15-29$ & Gravemente disminuido \\
G5 & $<15$ & Fallo renal
\end{tabular}

\begin{tabular}{lll}
\hline Categoría & Cociente albúmina/creatinina & Descripción \\
\hline
\end{tabular}

\begin{tabular}{lll}
\hline A1 & $<30$ & Normal a ligeramente elevada \\
A2 & $30-300$ & Moderadamente elevada \\
A3 & $>300$ & Muy elevada
\end{tabular}


Figura 1: Porcentaje de aciertos y fallos en el cuestionario de conocimientos antes y después de la EPR.
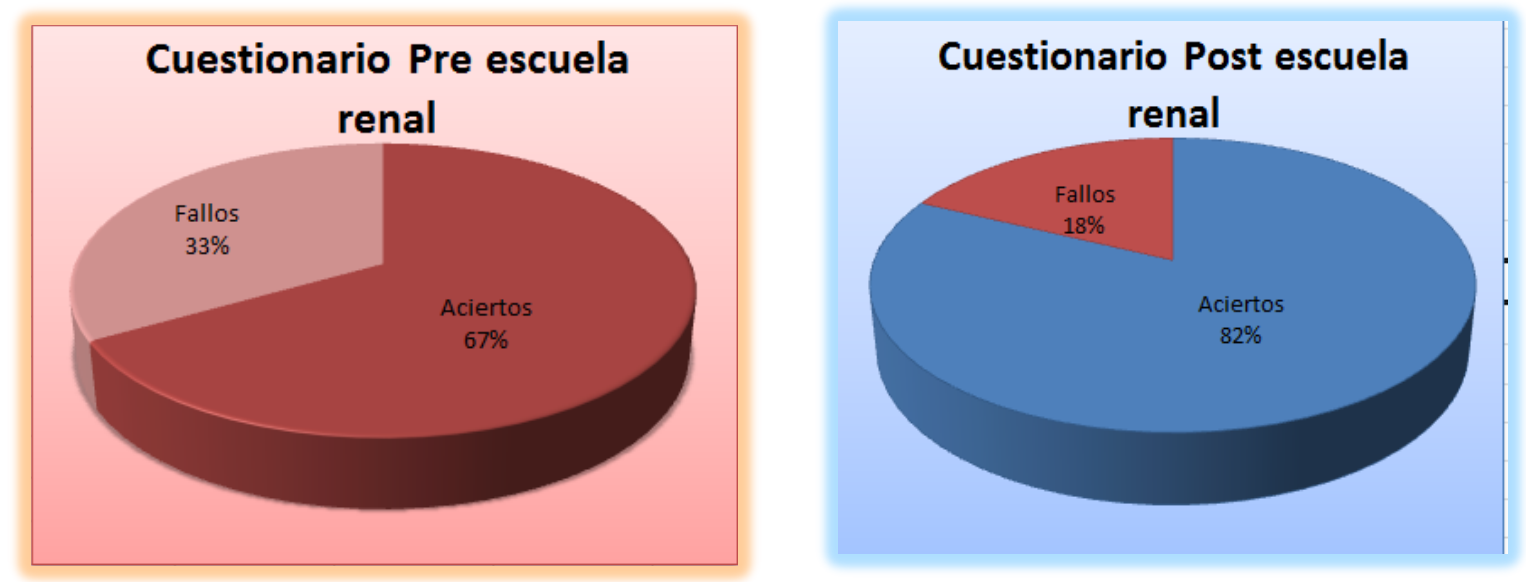